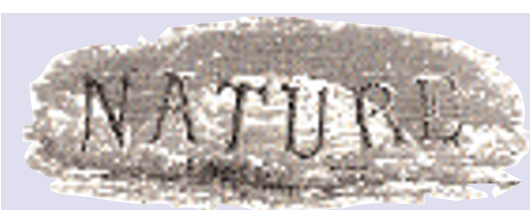

100 YEARS AGO

In "Monkeys; their Affinities and Distribution," by $\mathrm{Dr}$. A. R. Wallace, the author gives as one of the characters in which man differs from all the monkey tribe - "the perfect freedom of the hands from all part in locomotion." My object in writing this is to point out the peculiar way in which the majority of people move their arms and hands when walking or running. One may safely say that everybody, adults and children, at one time or another exercise this movement. The natural way in which children run is to "paddle" with the arms and hands, though trained runners do not do so. Now is it not possible that this muscular movement of the fore-limbs in opposite directions in the act of locomotion is a survival of the four-legged mode of progression of man's remote ancestors?... I believe that this theory has been thought of before, but I am unable to find any trace of it in the books I have consulted. I should be very grateful if any of your readers would enlighten me on the subject. Basil W. Martin From Nature 28 November 1901.

\section{YEARS AGO}

The story of the rabbit in Australia is so well known, so familiar an instance of the spread of an innocent introduction to plague dimensions, that fresh turns in its course must be spectacular to become news... The recognition that the virus disease, myxomatosis, might be an agent in reducing rabbit numbers directly invoked scientific enquiry and although early investigations... established that the disease could work, its effectiveness was small because of its very limited powers of spreading naturally through rabbit populations and groups... But as [this report] was prepared, a spectacular development occurred in the development of myxomatosis epidemics. It was known that the disease could be spread from sick to healthy rabbits by certain mosquitoes. As mentioned above, the first attempts to establish the disease in experimental sites were unsuccessful, but by continuing the introduction into warren colonies in the Murray River valley into the summer months of 1950-51, conditions favourable to success were encountered and mosquitoes rapidly spread the disease, so that by February 1951 the disease was reported widely in the Murray River system and its incidence had extended northwards along the MurrumBridgee, the Lachlan and the Darling Rivers, almost up to the Queensland border. From Nature 1 December 1951. we fully understand the mechanisms behind microbial control of carbon cycling. Given our current state of ignorance, any largescale manipulation of the oceans, such as proposals to increase phytoplankton growth by iron fertilization, could have catastrophic consequences $^{13,14}$. There is an urgent need for oceanographers to embark on a bold exploration of the oceans - this time at the millimetre scale.

Farooq Azam and Richard A. Long are in the Marine Biology Research Division, Scripps Institution of Oceanography, University of California at San Diego, La Jolla, California 93092-0202, USA.

e-mail:fazam@ucsd.edu

1. Azam, F. Science 280, 694-696 (1998).

2. Bidle, K. D. \& Azam, F. Nature 397, 508-512 (1999).
3. Kiørboe, T. \& Jackson, G. A. Limnol. Oceanogr. 46, 1309-1318 (2001).

4. Alldredge, A. L. \& Gotschalk, C. C. Cont. Shelf Res. 10, 41-58 (1990).

5. Mitchell, J. G., Pearson, L. \& Dillon, S. Appl. Environ. Microbiol. 62, 3716-3721 (1996)

6. Blackburn, N., Fenchel, T. \& Mitchell, J. Science 282, 2254-2256 (1998).

7. Grossart, H.-P., Riemann, L. \& Azam, F. Aquat. Microb. Ecol. 25, 247-258 (2001)

8. Mitchell, J. G., Pearson, L., Dillon, S. \& Kantalis, K. Appl. Environ. Microbiol. 61, 4436-4440 (1995).

9. Smith, D. C., Simon, M., Alldredge, A. L. \& Azam, F. Nature 359, 139-142 (1992).

10. del Giorgio, P. A. \& Cole, J. J. Annu. Rev. Ecol. Syst. 29, 503-541 (1998).

11. Martinez, J. et al. Aquat. Microb. Ecol. 10, 223-230 (1996). 12. Young, W. R., Roberts, A. J. \& Stuhne, G. Nature 412, 328-331 (2001).

13. Fuhrman, J. A. \& Capone, D. G. Limnol. Oceanogr. 36, 1951-1959 (1991)

14. Chisholm, S. W., Falkowski, P. G. \& Cullen, J. J. Science 294 309-310 (2001).

Cell cycle

\title{
Six steps to destruction
}

James E. Ferrell Jr

\section{Cell division relies on the properly timed activation and destruction of certain regulatory proteins. New work shows that many rounds of phosphorylation can help to establish the timing of protein destruction.}

T he process by which cells multiply consists of a strictly ordered series of events. A cell copies its genetic material, grows, and segregates the duplicated DNA into two new cells. Then, after a rest period, the process begins again. If cells are to progress smoothly from one phase of this 'cell cycle' to the next, key regulatory molecules must be turned on and off at just the right times. On page 514 of this issue, Nash and colleagues ${ }^{1}$ provide new insight into the molecular controls that allow budding yeast cells to move from the G1 phase (the rest period) to the $S$ phase (when DNA is copied). Their work builds on the fact that the Sic1 protein needs to be enzymatically modified with phosphate groups (phosphorylated) over and over again before cells can progress from G1 into the S phase. Sic1 provides a splendid example of how cells can use cumulative, multistep modifications to produce a strict, switch-like transition.

The job of the Sicl protein is to inhibit the protein complex that drives budding yeast cells from the G1 phase to the S phase $\mathrm{e}^{2-4}$. This protein complex comprises an enzyme from a family known as the cyclin-dependent kinases (this enzyme is Cdc28 in yeast, and Cdk1 in mammals) and its regulatory subunit, a cyclin protein (from the Clb family in yeast). During the G1 phase, cells have high levels of Sic1; this ensures that $\mathrm{Cdc} 28-\mathrm{Clb}$ complexes are inactive, so DNA replication is suppressed. At the end of the G1 phase, Sic1 is degraded, allowing $\mathrm{Cdc} 28-\mathrm{Clb}$ to drive cells into $S$ phase. In yeast strains that lack

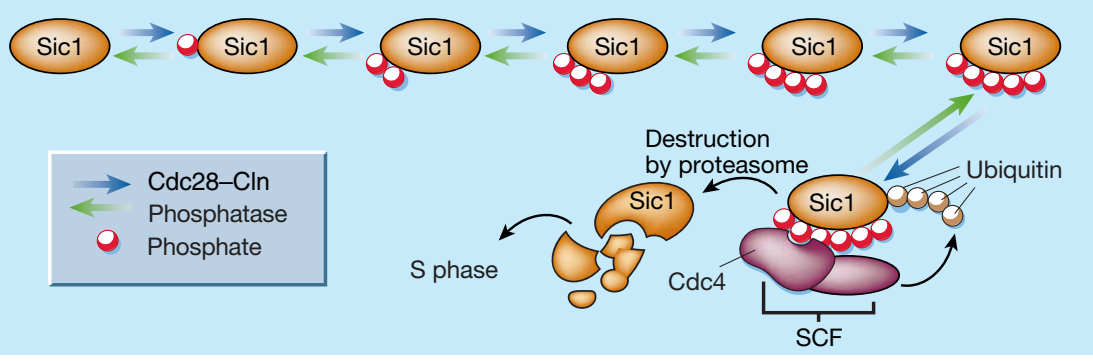

Figure 1 How cells get from the G1 phase to the S phase in their division cycle. The shift from G1 to $S$ phase is blocked by the protein Sicl, which inhibits a key enzymatic complex. Nash et al. ${ }^{1}$ have discovered that Sicl must be phosphorylated at least six times by the Cdc28-Cln complex before it can bind to the Cdc4 protein, be tagged with ubiquitin groups, and destroyed. The multistep phosphorylation of Sic1 may function as a sort of primordial timing mechanism, ensuring that Sicl destruction is precisely timed so that the $S$ phase does not happen too early. 
Sic1, DNA replication occurs too early; in strains with forms of this protein that are hard to destroy, DNA replication is delayed. So the timing of Sic1 degradation helps to establish the timing of the G1/S transition.

Before Sicl can be degraded, however, it must first be phosphorylated. The enzymes that do this comprise Cdc28 (again) and a Cln protein (another cyclin), and are activated in the G1 phase $e^{5,6}$. Phosphorylation allows Sic1 to bind to the protein $\mathrm{Cdc} 4$, which is a component of yet another protein complex, called $\mathrm{SCF}^{7,8}$. SCF in turn ensures that $\mathrm{Sic} 1$ becomes modified with a small peptide, ubiquitin, and targeted to the cellular protein-degrading machinery, the proteasome. The timing of Sicl destruction therefore depends on the timing of its phosphorylation.

Sicl has nine different sites that fit the 'consensus' for phosphorylation by a cyclindependent kinase (the consensus is the amino-acid sequence serine-proline or threonine-proline), and most, if not all, of the sites appear to become phosphorylated in $v i v o^{6}$. This raises the question of why Sicl has so many phosphorylation sites and is phosphorylated so many times.

Nash and colleagues ${ }^{1}$ approached this question by mutating different numbers of phosphorylation sites to find out how many are needed for proper destruction of Sic1. They found that Sicl with two, three or even five sites could not bind Cdc4 in vitro, but Sicl with six, seven or nine sites could. Likewise, Sic1 with just two, three or five phosphorylation sites prevented yeast strains from multiplying - presumably because they arrested in the G1 phase - but strains containing Sicl with six, seven or nine sites divided normally.

Why isn't one phosphorylation (or even five) sufficient? One possibility is that Cdc4 has six phosphate-binding pockets. But quantitative binding studies and mutational analysis indicate that it has only one $e^{1}$. An alternative is suggested by the consensus amino-acid sequence within phosphorylated peptides that allows them to bind optimally to Cdc4 (ref. 1). The mammalian cyclin E protein, another SCF substrate, has a single phosphorylation site that targets it for degradation; the sequence around this site fits the Cdc4-binding consensus and binds Cdc4 with high affinity. But it turns out that none of the phosphorylation sites in Sicl matches this consensus very well. This implies that Sicl needs to be phosphorylated many times because none of its phosphorylation sites is all that good at binding Cdc4.

But is it important that Sicl's destruction is driven by the combined effects of six phosphorylation sites? Or would a single high-affinity site work just as well? To find out, Nash et al. replaced seven of Sicl's nine phosphorylation sites with a single high- affinity site derived from cyclin $\mathrm{E}$. The resulting protein inhibited $\mathrm{Cdc} 28-\mathrm{Clb}$ in vitro, but failed to restrain DNA replication properly in vivo. Apparently it really does matter how Sic1 comes to be destroyed.

The fact that Sicl does not appreciably bind Cdc4 and begin to be destroyed until the sixth phosphate is added has several kinetic implications. It means that there should be a discrete lag time between the activation of Cdc28-Clns and the destruction of Sic1 (Fig. 1). Those ineffectual first five phosphorylations need to be accomplished before the critical sixth one can occur. So the time required for the first five phosphorylations acts as a temporal threshold for Cdc28-Clb activation. In one sense, the first five phosphorylations accomplish nothing - but sometimes doing nothing is of the utmost importance.

The requirement for six phosphates also means that the destruction of Sicl could be a highly nonlinear function of the amount of Cdc28-Cln activity in a cell. If so, the regulation of the G1/S transition would have a noise filter built into it - low levels of Cdc28-Cln activity would result in very little Sic1 being destroyed. Sic1 would then respond to higher levels of Cdc28-Cln activity in a decisive, switch-like fashion; doubling Cdc28-Cln activity could increase the destruction of Sicl by a factor of $2^{6}$ $(64 \text {-fold })^{9}$.

The six phosphorylations may also help to ensure the specificity of Sic1 destruction. Suppose that some kinase other than Cdc28-Cln can inappropriately phosphorylate $\operatorname{Sic} 1$ at a low frequency, $\epsilon$. The frequency of inappropriate destruction of Sic1 would then be as low as $\epsilon^{6}$. This property has been termed 'kinetic proof-reading' ${ }^{10}$ (P. Swain and E. Siggia, personal communication), and is another potential advantage of the six-phosphate mechanism.

Not all substrates of the SCF complex need multiple phosphorylations for destruction; cyclin E is a good example of this. So the timing and abruptness of destruction seems to be regulated by the properties both of the enzymes that catalyse phosphorylation and destruction, and of their substrates. SCF substrates apparently have a primordial timing mechanism built into them, on top of which other controls can be built.

Studies of budding yeast have a long history of yielding valuable insights into cell biology. Nash and co-workers ${ }^{1}$ have shown that such studies can also shed light on the biochemical logic of cell regulatory systems - with a lot of hard work, a bit of quantitative reasoning, and the ability to count to six.

James E. Ferrell Jr is in the Departments of

Molecular Pharmacology and Biochemistry,

Stanford University School of Medicine, Stanford,

California 94305-5174, USA.

e-mail: james.ferrell@stanford.edu

1. Nash, P. et al. Nature 414, 514-521 (2001).

2. Schwob, E., Bohm, T., Mendenhall, M. D. \& Nasmyth, K. Cell 79, 233-244 (1994)

3. Mendenhall, M. D., al-Jumaily, W. \& Nugroho, T. T. Prog. Cell Cycle Res. 1, 173-185 (1995).

4. Nugroho, T. T. \& Mendenhall, M. D. Mol. Cell. Biol. 14, 3320-3328 (1994).

5. Schneider, B. L., Yang, Q. H. \& Futcher, A. B. Science 272, 560-562 (1996)

6. Verma, R. et al. Science 278, 455-460 (1997).

7. Feldman, R. M., Correll, C. C., Kaplan, K. B. \& Deshaies, R. J. Cell 91, 221-230 (1997)

8. Skowyra, D., Craig, K. L., Tyers, M., Elledge, S. J. \& Harper, J. W. Cell 91, 209-219 (1997).

9. Ferrell, J. E. Jr Trends Biochem. Sci. 21, 460-466 (1996)

10. Hopfield, J. J. Proc. Natl Acad. Sci. USA 71, 4135-4139 (1974).

High-energy astrophysics

\section{A new spin on black-hole masses}

Charles Bailyn

The extreme environment surrounding a black hole provides an ideal test bed for the predictions of general relativity. New observations of a spinning black hole push current theories to their limits.

0 ne of the most enigmatic X-ray sources in our Galaxy is the prosaically named GRS1915 + 105, a type of binary star system known as a microquasar. It is of unique importance in high-energy astrophysics because it is the only microquasar that has remained active for more than a few months. Since its discovery ${ }^{1}$ in 1994, there have been hundreds of papers describing and interpreting its $\mathrm{X}$-ray, infrared and radio emission. Only the optical astronomers have been left out of the fun because a thick layer of galactic dust blocks optical light emitted by the source. The lack of optical data is unfortunate, because optical obser- vations (at visible and short infrared wavelengths) are usually required to provide crucial information about binary star systems, such as the orbital period and masses of the companion stars. On page 522 of this issue, Greiner et $a$. $^{2}$ at last report infrared observations of GRS1915 + 105 that enable them to determine these basic parameters. The results confirm the nature of the two companions - one is a mature Sun-like star, the other a spinning black hole — but the mass of the black hole gives an unexpected twist to the story.

Much of the excitement generated by GRS1915 + 105 is due to its 'superluminal' 